\title{
Holographic interferometry as a method to study conformational changes in macromolecules
}

\author{
Y. Barabash ${ }^{1}$, V. Kharkyanen' ${ }^{1}$ M. Zabolotny ${ }^{2}$, N. Sokolov ${ }^{3}$ \\ 1) Institute of Physics, NAS of Ukraine, 46 prospect Nauky, 03650 Kyiv-39, Ukraine; \\ 2) T.Shevchenko University, 64 Volodymyrs'ka vul., 04070 Kyiv, Ukraine; \\ 3) UKMA University, 2 Scovorody vul., 04070, Kiev, Ukraine \\ Phone: 38(044)570 7823.E-mail: ybar@mail.kar.net
}

\begin{abstract}
This paper concerns with our measurements of reaction center (RC) volume changes in the course of photoactivation using the method of holographic interferometry. As a principal value, we studied changes of a solution refraction index $(\Delta n)$ influenced by the sample volume expansion due to temperature changes $\left(\Delta n_{T}\right)$, light absorption $\left(\Delta n_{a b s}\right)$ and changes in the volume of photoactive molecules $\left(\Delta n_{V}\right)$. Using the scheme of temperature compensation we could reduce the task to the case $\Delta n_{T}<<\Delta n_{a b s}, \Delta n_{V}$. At RC concentrations close to $10^{15} \mathrm{~cm}^{-3}$, our experimental setup enabled to register the shift of an interference pattern up to 0.1 of the bandwidth. Our estimations for the case of a protein solution yields in relative changes in the molecular volume within $10^{-3} \ldots 10^{-2}$.
\end{abstract}

_Keywords: photoinduced change, electron transfer, holographic interferometer, photothermoplastical registering media, refraction index.

Paper received 08.04.02; accepted for publication 10.12.02.

\section{Introduction}

Earlier [1], we have studied in detail the slow kinetics of charge separation in the RC. Also, the role of conformational rearrangements in forming a stable "light" structural state of the $\mathrm{RC}$ has been elucidated by computer modeling. Together with literature data [1], these results make it possible to discuss the functionality of RC structural changes. However, the data on electron transfer cannot directly prove the character and mechanisms of $\mathrm{RC}$ structure changes. In what follows we describe our measurements of the RC volume changes in the course of photoactivation, using holographic interferometry methods. In many case holographic interferometry turns out to be a preferable, sometimes the only possible, technique to study the changes in the sample state $[2,3]$. In this paper, a study of conformational changes in the molecular complex of the $\mathrm{RC}$ is performed by the holographic interferometer using photothermoplastical registering media, that are reversible and do not require wet development. Optimization of operation conditions on all stages of hologram recording is realized using a control microprocessor device (CMD), including the control microprocessor and executive units with feedback elements.

\section{Experimental}

The schematic of the optical setup is shown in Fig.1.

The protein-pigment complex of the $\mathrm{RC}$, isolated from photosynthetic membranes of Rhodobacter Sphaeroides' cells using detergents, have been used as the object of the study. The RC were placed into a $0.01 \mathrm{M} \mathrm{Na-phosphate}$ buffer with $\mathrm{pH} 7.2$ that contained $0.05 \%$ of the detergent. Concentration of the RC molecules was $\sim 10^{-7} \mathrm{M}$. The size of the cuvette was $3 \times 5 \times 2.5 \mathrm{~cm}^{3}$, with walls of $1 \mathrm{~mm}$ thickness, and the cuvette consisted of two parts. The RC solution was placed into one half of the cuvette 8 , while the buffer of the same composition (the reference solution) was placed into the other part. The cuvette with both solutions was kept in the dark during 2 hours. After the hologram registration (registration time was $1 \mathrm{~s}$, the intensity of the object beam was $0.5 \mu \mathrm{W} / \mathrm{cm}^{2}$, the intensity of the reference beam was $50 \mu \mathrm{W} / \mathrm{cm}^{2}$ ), a phase shift of the object beam was introduced by rotating the plate 6 .

The interference pattern of bands was registered by a CCD camera connected to the PC. The accuracy of the interference bands position measurements was 0.025 of the interference band width. 


\section{Y. Barabash et al.: Holographic interferometry as a method to study...}

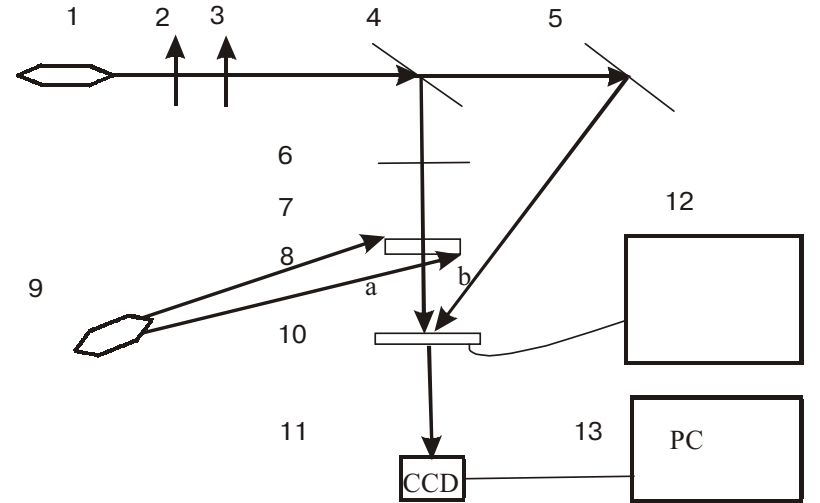

Fig.1. The schematic of optical setup: 1 - laser, 2,3 - collimators, 4 - semitransparent mirror, 5 - mirror, 6 - plane glass plate, 7 diffusive scatterer, $8 \mathrm{a}$ and $8 \mathrm{~b}$ - two cuvettes, with buffer and RC solution respectively, 9 - light source that lightens the cuvettes, 10 - plate with registering medium on it, $11-$ CCD camera, 12 hologram registration control block, 13 - computer.

\section{Results}

The results of experimental researches are summarized in Figs 2-5.

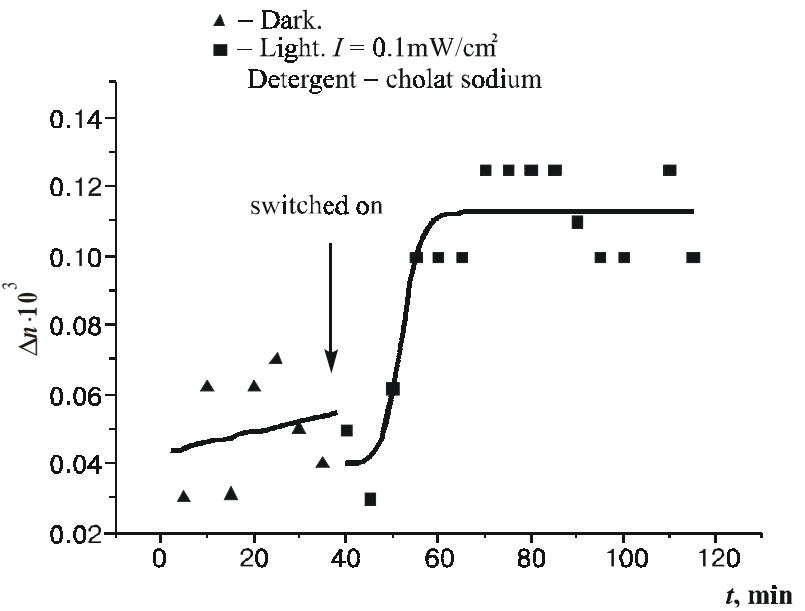

Fig. 2. Changes of the refraction index of the solution RCs with the ionic detergent of sodium cholat versus illumination time.

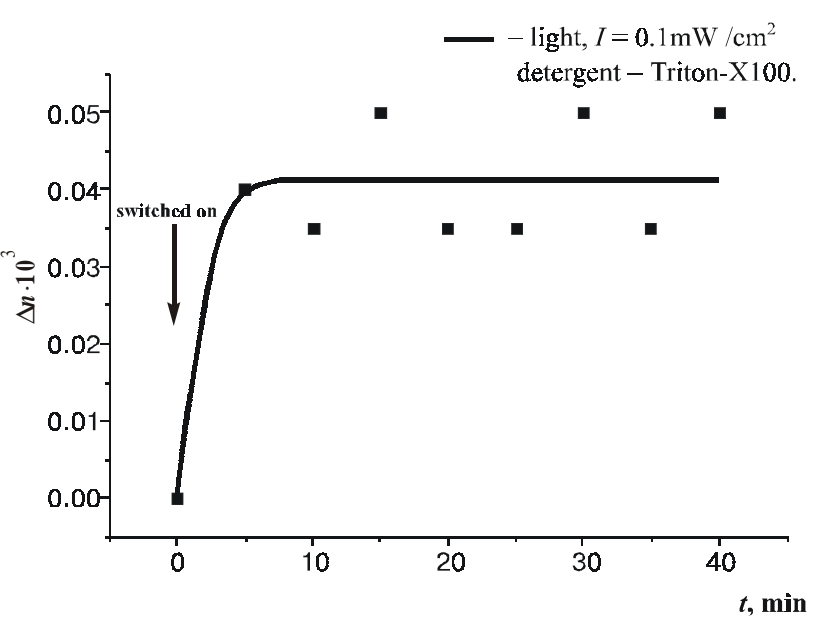

Fig. 3. Changes of the refraction index of the solution RCs with the non-ionic detergent Triton $\mathrm{X}-100$ versus illumination time.
Two grades of RC from Rb.sphaeroides, strain R-26, distinguished from each other only by type of the applied detergent were researched at their infusion from a membrane. In a Fig. 2 the dates of change of an index of refraction are shown at illumination of solution $\mathrm{RC}$, of ionic detergent, obtained using, cholat of a sodium In a Fig. 3, the data of change of refraction index are shown at illumination of the $\mathrm{RC}$ solution, obtained using nonionic detergent Triton X-100. For comparison of these data, with those of electronic transport, the study of kinetics for absorption spectra of these $\mathrm{RC}$ at the wavelength $865 \mathrm{~nm}$ were carried out irradiating RC solutions by light with $\lambda=865 \mathrm{~nm}$ with usage of solid-state light sources [4]. The results of such measurements are submitted in Figs 4,5. The Fig. 4 submits data on kinetics of the spectrum for RC obtained using the detergent cholat of a sodium, while Fig. 5, for RC obtained using the detergent Triton X-100.

\section{Discussion}

At the beginning, after registration of an interferogram of a dish, the interference bands cover the maps of both

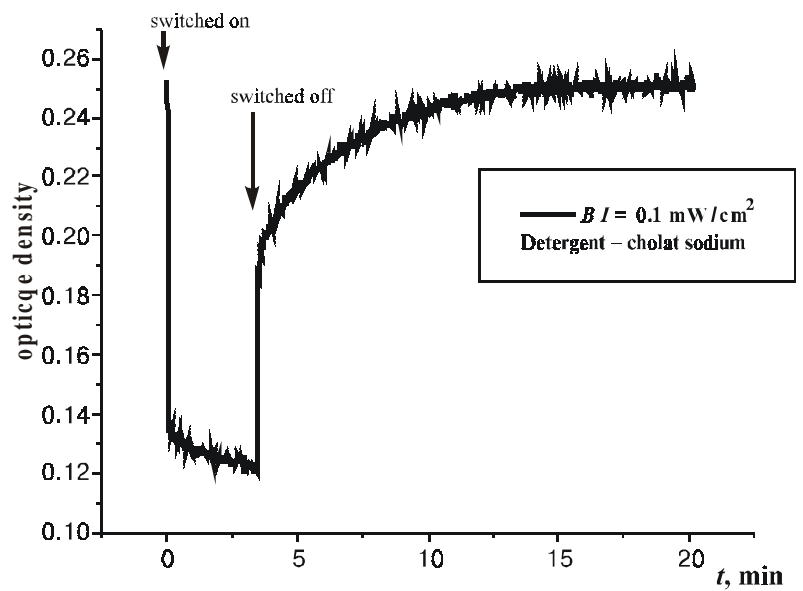

Fig. 4. Changes of the optical solution density of the RCs with the sodium cholat versus illumination time.

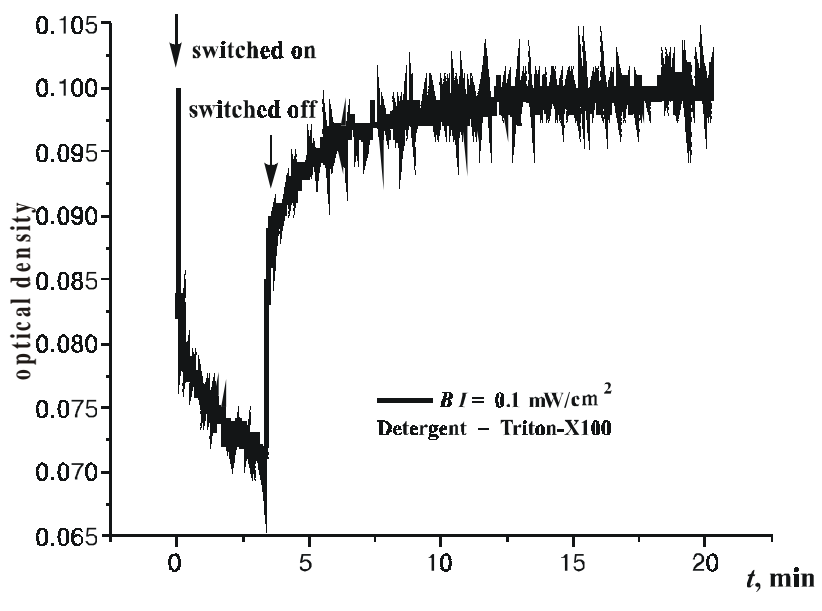

Fig. 5. Changes of the optical solution density of the RCs obtained using detergent Triton X-100 versus illumination time. 


\section{Y. Barabash et al.: Holographic interferometry as a method to study...}

parts of a dish without a relative displacement. With the course of time, at further illumination of a dish the energizing by light arose a relative displacement of interference bands between two parts of a dish. The displacement of interference bands inhering by that a part of a dish, in which the solution RC was, is directed downwards. Such direction of displacement of bands corresponds (meets) to increase of optical density of solution $\mathrm{RC}$. It makes $\sim 0.1$ bandwidths for $\mathrm{RC}$, chosen using cholat of sodium, and $\sim 0.05$ bandwidths for $\mathrm{RC}$, chosen using the detergent Triton X-100. Matching of Figs 2 and 3 displays that $\mathrm{RC}$ chosen using the detergent Triton $\mathrm{X}-100$ have shorter characteristic time of refraction index changes, than RC chosen using cholat of sodium.

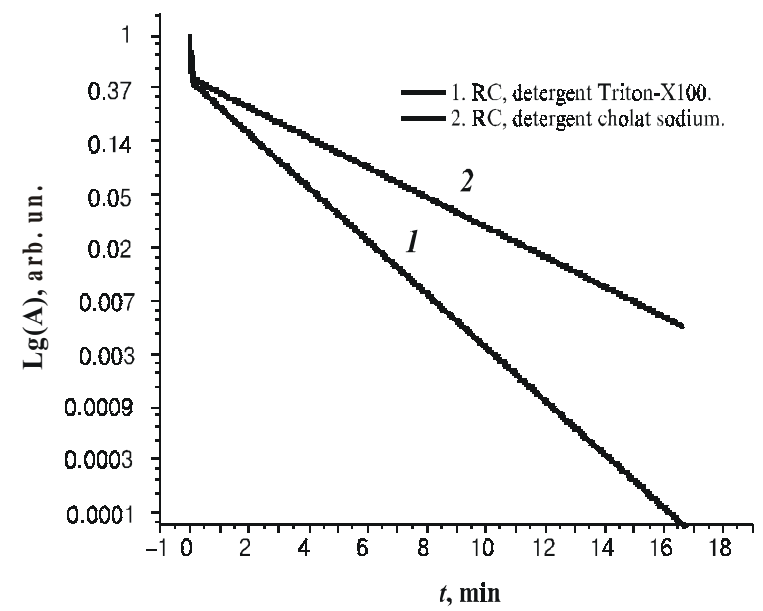

Fig. 6. The change of the optical solution density of the RCs obtained using detergent Triton X-100 (I) and the sodium cholat (2) versus illumination time.

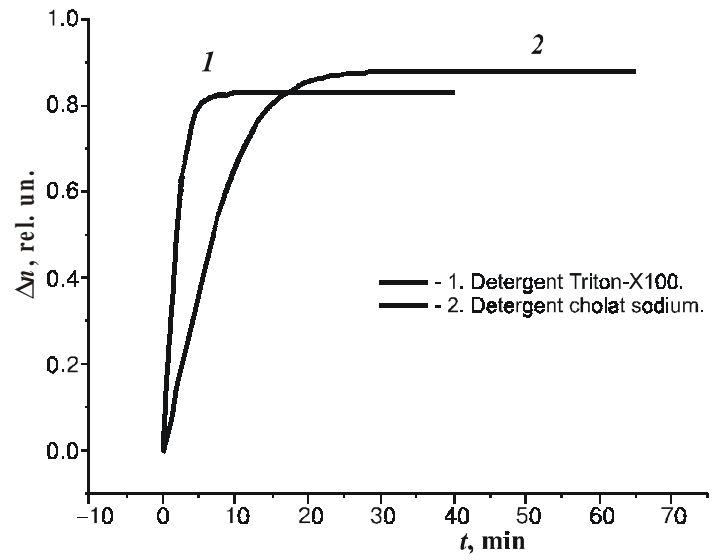

Fig. 7. Changes of refraction indexes for both RC types.

In a Fig. 6 the treated dates (Fig. 4,5) indicating restoring of absorption spectrums RC on $\lambda=865 \mathrm{~nm}$, after cutoff of energizing of light, are reduced. It is visible that in RC obtained using cholat of sodium, the restoring of the electronic transport goes more slowly, than in RC obtained using Triton X-100 (curves 2 and 1). In Fig. 7 the treated data (Figs 2,3) upon changes of refraction indexes for both types of RC are summarized for the case of their illumination. These graphs also display that the change of an index of refraction in $\mathrm{RC}$, obtained using cholat of sodium, goes more slowly than in that obtained using Triton X-100 (curves 2 and 1). Thus, the quality concurrence of a kinetics of restoring of absorption spectra to change of an refraction indexes of both RC types is observed during their illumination. Usually, a sluggish kinetics of RC absorption spectra is related to changes in their structure [4]. Therefore, it is necessary to evaluate the possible contribution of conformation changes to changes of a refraction index of $\mathrm{RC}$ solutions at their illumination. Let's consider a series of the factors influential in an offset value of interference bands. The displacement $\Delta$ of interference bands depends on change of refraction index $\Delta n$ of examined solution. Estimation of contributions into refraction index changes As is known $[2,3]$, the change $\Delta n$ in the refraction index can be written as:

$\Delta n=\Delta n_{T}+\Delta n_{a b s}+\Delta n_{V}$

where $\Delta n_{T}$ is caused by volume expansion of the sample due to temperature changes, $\Delta n_{a b s}$ - by light absorption, and $\Delta n_{V}$ - by changes in the volume of photoactive molecules.

Let $N$ stands for the number of bands of the interference pattern that is shifted. Then

$N=\frac{1}{\lambda} d \Delta n$

where $\lambda$ is the light wavelength, $\Delta n$ is refraction index change, $d$ is sample (cuvette) thickness. From (1) it follows that $\Delta n \sim 10^{-5}$ entails a change by unity in the band number.

Let us estimate temperature change $\Delta T$ due to illumination with exciting light of a power density $P$ during time $\tau$. Let the fraction of incident light absorbed by the sample be $h ; c$ stands for specific heat of the sample, $d-$ for its thickness. Then

$\Delta T=\frac{\eta P \tau}{C d \rho}$

With the parameters used, $P \approx 10^{-3} \mu \mathrm{W} / \mathrm{cm}^{2}, \tau=20$ $\min , \eta \cong 0.1, d \cong 2.5 \mathrm{~cm}, c=4000 \mathrm{~J} / \mathrm{kg} \cdot \mathrm{K}, \rho \cong 10^{3} \mathrm{~kg} / \mathrm{m}^{3}$, one has

$\Delta T \cong 0.01 \mathrm{~K}$

Assuming that the volume expansion coefficient $b$ of the solution is close to that of water, we find $\Delta n_{T}=$ $=-2.5 \cdot 10^{-6}$. The influence of ambient temperature can be estimated from the shift of the cuvettes interference bands with respect to the air and to each other while keeping the solution in the dark.

To estimate the influence of absorption spectra upon refraction index, we assume that the complex dielectric susceptibility $\varepsilon=\varepsilon^{\prime}+i \varepsilon^{\prime \prime}$ obeys the Kramers-Kronig rule and imaginary $-\mathrm{i} \varepsilon$ " parts of an inductivity of medium:

$$
\varepsilon^{\prime}\left(\lambda_{0}\right)=1+\frac{1}{\pi} P \int_{0}^{\infty} \frac{\varepsilon^{\prime \prime}(\lambda)}{\lambda-\lambda_{0}} d \lambda
$$


Integral (3) is understood in the sense of a principal value. It follows from the latter relation that the change $\Delta \varepsilon^{\prime}$ at the wavelength $\lambda_{0}$ is bound to the change of the imaginary part $\Delta \varepsilon$ " inductivities in all frequency band as follows:

$$
\Delta \varepsilon^{\prime}\left(\omega_{0}\right)=\frac{1}{\pi} P \int_{0}^{\infty} \frac{\varepsilon^{\prime \prime}(\lambda)}{\lambda-\lambda_{0}} d \lambda
$$

In its turn, the change of an imaginary part $\Delta \varepsilon^{\prime \prime}$ is bound to change of an exponent of absorption $\Delta \alpha$ at the same frequency by the following relation:

$\Delta \varepsilon^{\prime \prime}=\frac{n \cdot \Delta \alpha \cdot \lambda}{2 \pi}$

As is known [4], due to the radiation effect, the exponent of a sample absorption $\Delta \alpha$ varies in some frequency range $\left(\lambda_{\min }=230 \mathrm{~nm} ; \lambda_{\max }=1350 \mathrm{~nm}\right)$. The difference of absorption spectra is given in Fig. 8 .

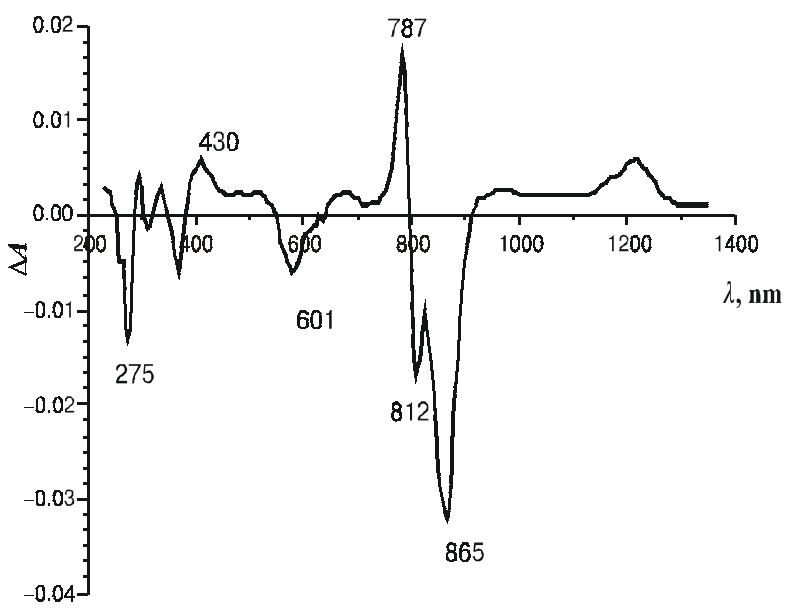

Fig. 8. The difference of absorption spectra of the RCs

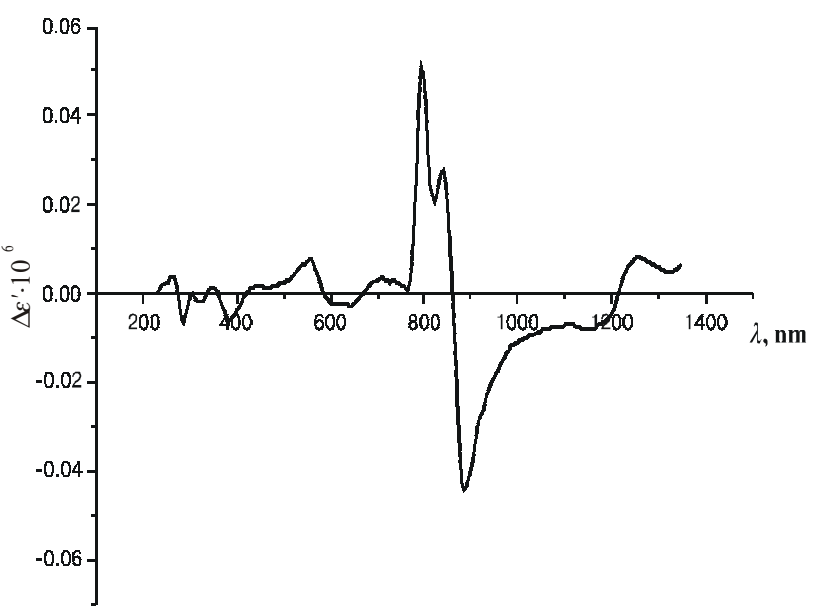

Fig. 9. The result of numerical integration of expression (6)
Therefore:

$\Delta \varepsilon^{\prime}\left(\lambda_{0}\right)=\frac{n \lambda_{0}}{2 \pi^{2}} P \int_{\lambda_{\min }}^{\lambda_{\max }} \frac{\Delta \alpha(\lambda)}{\lambda-\lambda_{0}} d \lambda$

In Fig. 9 the diagram of numerical integration of expression (6) is given. It is obvious from it, that $\left|\Delta \varepsilon^{\prime}\left(\lambda_{0}\right)\right| \leq 0.28 \cdot 10^{-8}$ and $\Delta \varepsilon^{\prime}\left(\lambda_{0}\right)$ is less than zero. In absorbing media the change of the refraction index is determined by a relation:

$\Delta n_{a b s}=\frac{1}{4 n}\left(\Delta \varepsilon_{1}+\frac{\varepsilon^{\prime} \cdot \Delta \varepsilon^{\prime}+\varepsilon^{\prime \prime} \cdot \Delta \varepsilon^{\prime \prime}}{\sqrt{\varepsilon^{\prime 2}+\varepsilon^{\prime \prime 2}}}\right)$

Now it is possible to calculate the retrieved value $\Delta \varepsilon^{\prime}\left(\lambda_{0}\right)$ and value $\Delta \varepsilon^{\prime \prime}\left(\lambda_{0}\right)$ defined via the relation (5), $\Delta n_{a b s}$ which is approximately equal to $-2 \cdot 10^{-8}$. So, the experimentally apparent change $\Delta n \sim 10^{-6}$ exceeds possible values $\Delta n_{a b s} \sim 10^{-8}$ and does not coincide in its sign both with $\Delta n_{T}$ and $\Delta n_{a b s}$. Therefore, the apparent changes of the refraction index can be caused only by a change of the specific volume of the dissolved RC molecules. The value of the relative change of molecular volume of molecules $\Delta V / V$ is determined by the change of the refraction index of the solution, its quantity and molar concentration X of molecular RC in the studied solution [5].

$\frac{\Delta V}{V} \geq \frac{\Delta n}{n \cdot \mathrm{X}}$

In the used RC solution $X \approx 5 \cdot 10^{-4}$, and $10^{-6} \leq \Delta n \leq 10^{-5}$. Therefore, the relative change of volume of $\mathrm{RC}$ molecules in a solution makes $10^{-3}-10^{-2}$.

\section{Conclusions}

1. The change of the refraction index of the $\mathrm{RC}$ solution occurs when illuminating it with light of $\lambda=630 \mathrm{~nm}$ and intensity of $10^{-3} \mathrm{~W} / \mathrm{cm}^{2}$. The change of the refraction index is not time-dependent for exposition times $t>20 \mathrm{~min}$. Therefore, the time of the transition processes does not exceed $20 \mathrm{~min}$ at this light intensity.

2. The changes in the $\mathrm{RC}$ solution temperature and in the dynamics of the electron transitions caused by light absorption do not affect the changes in the refraction index of the RC solution. The $\Delta n$ value depends on the conformational changes of the RC complex.

3 . The refraction index $n$ returns to its initial value after turning off the light. The photoinduced changes in the $\mathrm{RC}$ are reversible.

4. The photoinduced change in the $\mathrm{RC}$ molecular volume amounts to $0.1 \ldots 1 \%$ and can be caused by structural changes in the RC molecular complex (e.g. the angle between the bonds bacteriochlorophile, bacteriopheophetin - primary and secondary chinon acceptors). The high probability of such a process is due to the long lifetime of the nonequilibrum electron on the secondary chinon acceptor. 


\section{Y. Barabash et al.: Holographic interferometry as a method to study...}

\section{References}

1. Gousha A.O., Kharkyanen V.N., Scott G.N., Alfred R., Holzwarth A.R., Self-regulation phenomena in bacterial reaction centers // Biophysical Journal. 79, pp.1237-1252 (2000).

2. Schulenberg P.J., Gartner W., Braslawsky S.E. // J.Phys.Chem., 99, p. 9617 (1995).
3. Schulenberg P.J., Braslawsky S.E. "Progress in Photothermal and Photoacustic Science and Technology", // Spie Opt. Eng. Press, Bellingham, 58, Eds Manddeles A., Hess P.(1997).

4. Rubin A.B. Biophysics, vol.2. Ed by Mosc. University, M., p. 468 (2000).

5. Y.Barabash, V.Kharkyanen, M.Zabolotny, N.Sokolov, The study of conformational macromolecules of RC from RB Sphaeroides// Proceedings of the XV International SchoolSeminar "SPECTROSCOPY OF MOLECULES AND CRYSTALS" 23-30 June, Chernihiv, Ukraine, p.196 (2001). 